\title{
Unusual manifestations of brucellosis: a retrospective case series in a tertiary care Greek university hospital
}

I. Starakis, ${ }^{1}$ E.E. Mazokopakis ${ }^{2}$ and H. Bassaris ${ }^{1}$

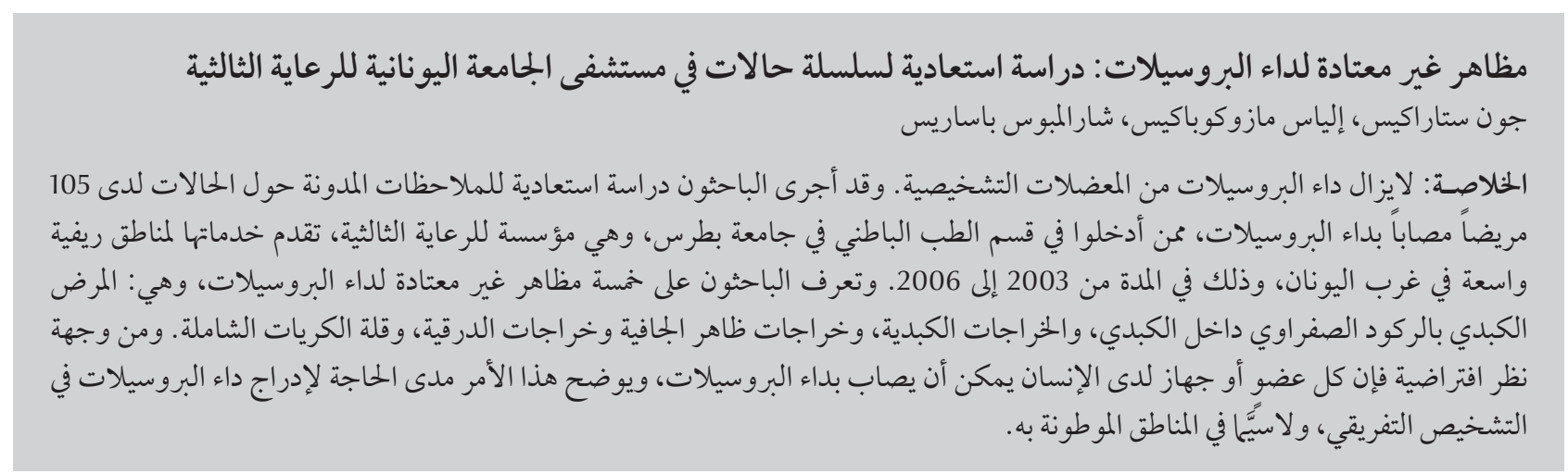

ABSTRACT Brucellosis remains a diagnostic puzzle. We retrospectively studied the case notes of 105 patients with brucellosis who were admitted in the Department of Internal Medicine of the University of Patras Hospital, a tertiary care institution serving an extended rural area in western Greece, from 2003 to 2006 . Five unusual causes of brucellosis were identified: hepatic, epidural and thyroid abscesses, intrahepatic cholestatic liver disease and pancytopenia. Virtually every human organ and system can be involved in brucellosis, which highlights the need to include brucellosis in the differential diagnosis, especially in endemic areas.

\section{Manifestations inhabituelles de la brucellose : série de cas rétrospective dans un hôpital universitaire de soins tertiaires en Grèce}

RÉSUMÉ La brucellose reste une énigme en termes de diagnostic. Nous avons étudié rétrospectivement les notes prises sur les cas de 105 patients atteints de brucellose, admis entre 2003 et 2006 dans le service de médecine interne de l'hôpital universitaire de Patras, établissement de soins tertiaires qui dessert une zone rurale étendue à l'ouest de la Grèce. Cinq causes inhabituelles de brucellose ont été identifiées : abcès hépatique, épidural et thyroïdien, cholestase intra-hépatique et pancytopénie. Quasiment tous les organes et systèmes de l'organisme peuvent être impliqués dans cette maladie, ce qui souligne la nécessité d'inclure la brucellose dans le diagnostic différentiel, en particulier dans les zones d'endémie. 


\section{Introduction}

Brucellosis continues to pose a human health risk worldwide, regardless of efforts to eradicate the disease from domestic animals. At present, Brucella melitensis represents the principal cause of human brucellosis in most countries, including Greece.

Brucellosis remains a diagnostic puzzle due to its ambiguous, non-specific manifestations and increasingly unusual presentations. The disease is one of the great "imitators" in the universe of infectious diseases, with its ability to mimic various other diseases. The most common sites involved are osteoarticular, especially sacroiliitis (20\%-30\%) with large joints most frequently affected in children, and the genitourinary system, especially epididymoorchitis (2\%-40\% of males). Unusual manifestations of brucellosis, with a prevalence rate lower than $5 \%$ of cases, are neurobrucellosis $(1 \%-2 \%)$ which is usually presents as meningitis, endocarditis (1\%) (mostly left-sided) and hepatic abscess (1\%). There are other exceptionally rare manifestations including thyroid, splenic and epidural abscess, pneumonitis, pleurisy or empyema and uveitis.

This retrospective case series assembles our recent experience of the diagnostic challenges in combating this protean disease in an endemic area of western Greece. From the 105 casenotes we studied, the 5 cases of brucellosis reported in this paper all had an atypical and misleading presentation and all constituted a life-threatening clinical situation. They highlight the need for vigilance by physicians and close collaboration with the microbiology department to swiftly diagnose and treat this infection, in order to avoid deleterious consequences. Laboratory testing is indispensable for diagnosis and should always be combined with a high index of clinical suspicion. Establishing a history of travel to endemic countries plus exposure to animals and/ or their products is crucial in order to reach an early clinical diagnosis.

\section{Case reports}

\section{Case 1}

A 67-year-old previously healthy farmer presented to our hospital in March 2003 with fever up to $40^{\circ} \mathrm{C}$, rigor, fatigue and abdominal pain of 5 days' duration. $\mathrm{He}$ mentioned frequent consumption of unpasteurized dairy products. On examination he looked moderately ill, and right quadrant tenderness along with a positive Murphy sign was noted.

Laboratory tests showed the following values: haematocrit $(\mathrm{Ht}) 36 \%$, white blood cell count (WBC) 4000 cells/ $\mathrm{mm}^{3}$ (neutrophils 60\%, lymphocytes $24 \%$, monocytes $12 \%$, basophils $3 \%$, eosinophils 1\%) and platelet count (PTL) $133000 / \mathrm{mm}^{3}$ (normal range: 150000 to $\left.450000 / \mathrm{mm}^{3}\right)$. Erythrocyte sedimentation rate (ESR) was $107 \mathrm{~mm}$ in 1 hour. Liver function tests were abnormal: aspartate aminotransferase (AST) $155 \mathrm{U} / \mathrm{L}$ (normal range: 5-40 U/L), alanine aminotransferase (ALT) $281 \mathrm{U} / \mathrm{L}$ (normal range: 5-40 $\mathrm{U} / \mathrm{L}$ ), gamma-glutamyl-transpeptidase $(\gamma \mathrm{GT}) 143 \mathrm{U} / \mathrm{L}$ (normal range: 10-75 U/L), alkaline phosphatase (ALP) 185 $\mathrm{U} / \mathrm{L}$ (normal range: 35-125 U/L) and lactate dehydrogenase (LDH) 680 U/L (normal range: 80-230 U/L). All other laboratory findings were within normal limits.

Abdominal ultrasonography revealed mild hepatomegaly. Brucella agglutination test was negative. The patient was empirically treated with ciprofloxacin $400 \mathrm{mg}$ every 12 hours and metronidazole $500 \mathrm{mg}$ every 8 hours. On day 4 his clinical condition worsened and a new ultrasound revealed an enlarged gall bladder with thickened wall, suggesting an empyema. Abdominal computerized tomography (CT) scanning demonstrated mild hepatosplenomegaly and a hepatic lesion with central necrosis, consistent with the abscess. The patient was transferred to the surgical ward and operated on the same day. A cholecystectomy and aspiration of the abscess were performed.

Two days later, B. melitensis was isolated from 2 blood cultures that had been drawn on admission. B. melitensis was also cultured from the aspirate of the liver abscess. Gallbladder biopsy revealed acute cholecystitis, but gallbladder culture was negative. Antibiotic treatment was changed to doxycycline $200 \mathrm{mg}$ /day, rifampicin $900 \mathrm{mg}$ /day and gentamycin $240 \mathrm{mg} /$ day. The patient was discharged on day 14 and was prescribed doxycycline $200 \mathrm{mg} /$ day with rifampicin $900 \mathrm{mg} /$ day for 3 months.

\section{Case 2}

A 41-year-old man with no past medical history presented in June 2003 and was admitted to our hospital complaining of acute low back pain of 10 days' duration. The pain progressively worsened and 2 days before admission he started to suffer fever with rigors, malaise and profuse night sweating. On physical examination, only low back tenderness was noted.

Laboratory tests showed the following values: Ht 43\%, WBC 19000 cells/ $\mathrm{mm}^{3}$ (neutrophils $83 \%$, lymphocytes $15 \%$, monocytes 2\%), PLT 450 000/ $\mathrm{mm}^{3}$, ESR $120 \mathrm{~mm} / \mathrm{h}$, C-reactive protein (CRP) level $11 \mathrm{mg} / \mathrm{dL}$ (normal range: $0.08-0.8 \mathrm{mg} / \mathrm{dL}$ ). All other laboratory findings were normal. The Wright agglutination test for Brucella was positive at titres of 1:320.

A magnetic resonance imaging (MRI) study was performed. On the pre-contrast T1-sagittal images, a collection of fluid with low signal intensity was detected in the posterior epidural space that was anteriorly compressing the dorsal sac. A decreased signal intensity of the L5 and S1 vertebral bodies was also demonstrated. Following gadolinium (Gd) administration, there 
was marked enhancement of the L5 and $\mathrm{S} 1$ vertebral bodies, the L5-S1 intervertebral disk and the epidural fluid collection, consistent with spondylodiscitis and abscess formation at the L5S1 level. While awaiting blood culture results, meropenem and vancomycin intravenously were administered along with rifampicin and doxycycline per os. The fever subsided the next day but the low back pain remained unchanged and was intensified when the patient's right leg was elevated at $35^{\circ}$.

On the 6th hospital day, blood cultures revealed B. melitensis. The patient was operated on the same day and a broad L5 laminectomy was performed with complete abscess removal and decompression of the S1 nerve root. B. melitensis was also isolated from the abscess. The patient's postoperative course was uneventful and he was discharged 7 days after surgery. Rifampicin $600 \mathrm{mg} /$ day and doxycycline $200 \mathrm{mg} /$ day were administered for 3 months.

\section{Case 3}

An 87-year-old woman suffering from mild hypertension and rheumatoid arthritis first presented in April 2004 and was urgently admitted to our hospital because of fever up to $38^{\circ} \mathrm{C}$, severe neck pain and difficulty swallowing solids and liquids of 12 days' duration. She was on oral methylprednisolone ( $8 \mathrm{mg}$ /day).

On physical examination the enlarged thyroid gland was very tender to palpation. There was a large swelling occupying the region of the thyroid gland, the overlying skin was warm and erythematous, but no fluctuation was apparent. Swallowing aggravated the pain. Blood and urine cultures were obtained and ticarcillin-clavulanate in combination with vancomycin was administered intravenously.

Laboratory evaluation demonstrated a leukocyte count of 5300 cells $/ \mathrm{mm}^{3}$ (60\% neutrophils, $27.7 \%$ lymphocytes, $8.5 \%$ mononuclear cells, $3.4 \%$ eosinophils and $0.4 \%$ basophils). Ht was
36\%, haemoglobin $(\mathrm{Hb}) 11.1 \mathrm{~g} / \mathrm{dL}$ and PTL count $190000 / \mathrm{mm}^{3}$; ESR was 106 $\mathrm{mm} / \mathrm{h}$ and blood biochemistry profile was normal. The Wright agglutination test for Brucella was positive at titres of 1:640. A computerized tomography of the thyroid revealed 2 cystic areas in the right lobe and the isthmus of the gland which did not enhance with contrast.

A fine-needle aspiration of the thyroid fluctuant mass was performed, and culture yielded B. melitensis. Blood cultures also isolated B. melitensis. Rifampicin $600 \mathrm{mg} /$ day and doxycycline $200 \mathrm{mg} /$ day were added to the antibiotic regimen. A detailed history revealed that the patient was occasionally consuming unpasteurized milk and fresh cheese. The patient's condition deteriorated over the next 2 days, with high fever and development of necrotic skin areas and sinus tracts over the fluctuant right lobe mass. She was operated on and the right thyroid lobe and isthmus were removed.

The patient had an uneventful course and was discharged 5 days after the operation. A barium esophagogram performed after her discharge failed to show a pyriform sinus fistula (PSF). Rifampicin $600 \mathrm{mg} /$ day and doxycycline $200 \mathrm{mg} /$ day were prescribed for 3 months, and doxycycline alone was administered for 6 more months.

\section{Case 4}

A 39-year-old shepherd presented to the emergency department in February 2005 and was admitted with fever up to $40{ }^{\circ} \mathrm{C}$, rigors, fatigue and abdominal pain of 7 days' duration. On examination, he looked unwell, and a diffuse abdominal tenderness was noted. Neither lymphadenopathy nor hepatosplenomegaly was noted.

Blood analysis on admission showed pancytopenia $(\mathrm{Hb} 9.1 \mathrm{~g} / \mathrm{dL}$, Ht $29.2 \%$, total WBC $1150 / \mathrm{mm}^{3}$ with a neutrophil count of $450 / \mathrm{mm}^{3}$, PTL $98000 / \mathrm{mm}^{3}$ and reticulocytes $0.2 \%$. Blood smear examination revealed non- specific findings. CRP level was elevated $(19.5 \mathrm{mg} / \mathrm{dL})$. Liver function tests were mildly elevated (AST $48 \mathrm{U} / \mathrm{L}$, ALT $52 \mathrm{U} / \mathrm{L}, \gamma \mathrm{GT} 75 \mathrm{U} / \mathrm{L}$ and LDH 343 $\mathrm{U} / \mathrm{L}$ ). All other laboratory findings were within normal limits.

Brucella agglutination test was negative. On day 4, bone marrow aspiration cultures and biopsy was performed. Bone marrow aspirate revealed hypocellularity, which was confirmed by bone marrow biopsy. Granulomas were not present in the bone marrow biopsy. He had a normal chest radiograph. Abdominal ultrasonography and CT scanning were normal.

The patient was empirically treated with meropenem $1 \mathrm{~g} \times 3$ and vancomycin $\lg \times 2$. B. melitensis was isolated from 2 blood cultures and bone marrow cultures. Antibiotic treatment was switched to doxycycline $200 \mathrm{mg} /$ day, rifampicin $900 \mathrm{mg} /$ day and gentamycin $240 \mathrm{mg} /$ day. Pancytopenia was resolved during treatment and the patient was discharged on day 14 , with doxycycline $200 \mathrm{mg} /$ day and rifampicin 900 $\mathrm{mg} /$ day for 2 months.

\section{Case 5}

A 50-year-old woman presented in October 2006 and admitted to our hospital on the same day because of low-grade fever of 10 days' duration, malaise, anorexia and sweating. She had an unremarkable medical history. Consumption of unpasteurized dairy products during the previous month was recorded.

Physical examination revealed mild hepatomegaly and splenomegaly, confirmed by an abdominal ultrasound and CT imaging. Laboratory evaluation showed: Ht $34 \%$, WBC 5700 cells $/ \mathrm{mm}^{3}$ (neutrophils 62\%, lymphocytes 28\%, monocytes $8 \%$, eosinophils $2 \%$ ), PLT $119000 / \mathrm{mm}^{3}$, ESR $85 \mathrm{~mm} / \mathrm{h}$, CRP $8.95 \mathrm{mg} / \mathrm{dL}$, ALT $527 \mathrm{U} / \mathrm{L}$, AST 692 U/L, GGT 455 U/L, ALP 437 U/L, bilirubin $4.7 \mathrm{mg} / \mathrm{dL}$, direct bilirubin 2.4 $\mathrm{mg} / \mathrm{dL}$, albumin $3.5 \mathrm{~g} / \mathrm{dL}$ and LDH 
492 U/L. Brucella agglutinin titre was positive at $1 / 640$. Blood cultures isolated B. melitensis on day 7 .

Doxycycline was administered (100 mg per os twice daily) for 6 weeks plus $1 \mathrm{~g}$ streptomycin intramuscularly for the first 21 days, beginning on the 3 rd day of hospitalization, while waiting for the serological and cultural confirmation. Liver dysfunction gradually improved and was finally completely restored 1 month after discharge.

\section{Discussion}

\section{Case 1}

Hepatic involvement in infections caused by Brucella spp. is common (in $50 \%$ of patients), but the production of single or multiple liver abscesses is exceptional, with an incidence of only $0.2 \%[1]$. A review of the medical literature showed only 40 cases from 1904 until now, almost all involving adults with chronic infection [2,3]. In most reported cases of brucellar abscess, fever is the main symptom. Two-thirds of patients had prolonged, slight pain in the right upper abdominal quadrant.

The most significant laboratory disturbances include increased ESR and normochromic, normocytic anaemia in half the patients, with no leukocytosis or leukopenia, and a normal or slightly neutrophilic differential count [4]. Liver function tests commonly portray an increase in cholestasis-dependent enzymes with values 4- to 5-fold above normal values in $50 \%$ of cases, generally with no increased bilirubin or transaminases.

Ultrasound most commonly shows a single, hypoechoic, lesion with $\geq 1$ centrally located calcium deposits [5]. CT findings most frequently portray a hypodense area, and often $\geq 1$ saccular, loculated forms, and $\geq 1$ calcifications [5]. The presence of central calcifications is a very common finding, particularly in long-standing involvement, which should alert the physician to the possibility of a brucellar abscess [4].

In our case, suppurative disease of the liver developed in the acute phase of the disease. This may be observed in prolonged, recurrent brucellosis, but is quite rare in acute brucellosis. The absence of calcium deposition in the liver abscess, a constant characteristic of hepatic suppurative complications in chronic brucellosis, was also confirmation of the acute form of the disease in our patient.

\section{Case 2}

Spinal epidural abscess (SEA) is a rare condition, which is difficult to diagnose, may be complicated by potentially disastrous neurological or vascular complications and can be fatal if left untreated. SEA accounts for 1-2 in every 10000 hospital admissions, and Staphylococcus aureus is the agent most frequently implicated [6]. Brucella spp. are responsible for only $0.1 \%$ of cases. Risk factors include immunocompromised states, but no predisposing conditions are found in $20 \%$ of patients. Skin abscesses and furuncles represent the most common source of infection. The most common presenting symptoms of SEA are back pain and fever, and the lapse between the onset of pain and neurologic deficits is quite varied. Neurologic dysfunction is often disproportionate to the observed degree of compression and a combination of compressive and ischaemic effects may act in synergy to produce the deleterious sequelae of epidural abscess. MRI has the greatest diagnostic accuracy and is the method of first choice in the diagnostic process [7]. Typical MRI findings in vertebral osteomyelitis include decreased signal intensity in the disk and adjacent vertebral bodies on T2-weighted images, loss of endplate definition on T1-weighted images and contrast enhancement of the disk, adjacent vertebral bodies and involved paraspinal and paravertebral soft tissues on T1-weighted images [8].
The therapeutic method of choice is laminectomy and drainage of the abscess combined with antibiotics.

\section{Case 3}

Infections of the thyroid are rare because the gland is resistant to infection. Hendrick reported that only 5\% of 117 reviewed patients with thyroiditis finally developed an abscess [9]. Infectious thyroiditis may be either acute or chronic. Acute suppurative thyroiditis (AST) can lead to abscess formation if left untreated, and is usually caused by Gram-positive or Gram-negative organisms; S. aureus predominates, but pneumococcal, Salmonella spp., mycobacterial, parasitic, fungal and pneumocystic infections may also occur. AST most likely occurs in patients with pre-existing thyroid disease, with congenital anomalies such as a PSF, with immunosuppression, with the acquired immunodeficiency syndrome (AIDS) and in elderly or debilitated patients. AST usually presents with pain, tenderness and fever. Sore throat, dysphonia and dysphagia may also be present. Haematogenous seeding from a distal site of infection may be the most common cause of thyroid infection, although the precise infectious source is often unidentified [10].

Laboratory features include elevated ESR and possibly an elevated WBC with a left shift. Radioactive iodine scanning may be normal or show suppurative areas appearing as "cold" nodules. Ultrasound and CT scanning may demonstrate the abscess. Most authorities agree that a barium oesophagogram should be done after the infective process subsides to look for an occult PSF [11]. In these cases, reconstructive surgery is essential to prevent recurrence of the abscess. The essential steps in evaluation are fineneedle aspiration biopsy, with Gram staining and culture of the thyroid to identify the causative organism. Surgery is the most frequently recommended 
management of a thyroid abscess, either excision or drainage, combined with suitable parenteral antibiotic treatment, although it has been suggested that thyroid abscesses can be treated with antibiotics alone and surgery should be employed only if antibiotics are unsuccessful [12].

\section{Case 4}

Brucellosis is related to an assortment of nonspecific haematological abnormalities. Akdeniz et al. documented anaemia in $55 \%$ of their patients, leukopenia in $21 \%$, thrombocytopenia in $26 \%$, and pancytopenia in $8 \%$ [13]. The pathogenesis of pancytopenia is poorly understood, but hypersplenism [14], haemophagocytosis [15], and granulomatous lesions of the bone marrow [16] seem to beimplicated. Haemophagocytosis has been documented in 10\%-30\% of patients with brucellosis, and in $87 \%$ of patients with pancytopenia caused by this disease [16]. Pancytopenia is not a rare manifestation, with an incidence varying from $2 \%$ to $21 \%$ and bone marrow aspiration specimens usually reveal normo- or hypercellularity [17].

In this case, the patient presented only with febrile pancytopenia, demonstrating bone marrow hypoplasia, which is a rare feature of brucellosis. There was no haemophagocytosis or granulomatous infiltration of the bone marrow. Hepatosplenomegaly and bone marrow hyperplasia, indicating that pancytopenia may be due to hypersplenism [18], were also absent in our patient. Physicians should always keep in mind the possibility of brucellosis in febrile neutropenic patients or those presenting with aplastic anaemia, especially if the patients are living in, or have travelled to, endemic areas.

\section{Case 5}

Brucellosis involves the liver in different modes. Hepatitis is frequent, usually manifested as a mild increase of transaminases, while liver abscess or jaundice are uncommon [19]. Ascites may be present, either as a temporary exacerbation of pre-existing hepatic disease or as frank peritonitis [20]. Asymptomatic, mild cholestatic hepatitis accounted for approximately $16 \%$ of acute cases of brucellosis in a small study from Greece [21]. Granulomas can be present in liver-biopsy specimens in cases of both B. melitensis and B. abortus. Akritidis et al. demonstrated granuloma development in the liver parenchyma in all patients and in the portal spaces in a majority of patients [22]. Varying degrees of cellular infiltration of parenchymal tissue and portal spaces, giant cells in granulomas, parenchymal necroses and Kupffer cell hyperplasia were also noted.

\section{Conclusions}

Virtually every human organ and system can be involved in brucellosis, a fact that emphasizes the importance of always including brucellosis in the differential diagnosis, especially in endemic areas, even if the clinical aspects are not entirely consistent with it.

Although liver abscess formation is rarely observed in the chronic stages of brucellosis, acute cholecystitis and suppurative liver disease may be the main manifestations during the acute phase.

Brucella infection should be considered in patients presenting with signs and symptoms of spinal epidural abscess, a rare but devastating condition if not diagnosed early and treated promptly and effectively.

Infections of the thyroid are quite rare, but progress to abscess formation may occur, with all the deleterious consequences of advanced suppuration in the neck. Brucella spp. are very rarely implicated in the pathogenesis of this disease, but should be considered as the etiologic agent especially in elderly, debilitated patients.

Brucellosis may present as febrile neutropenia or even as aplastic anaemia, and bone marrow cultures are the gold standard in isolating the organism.

Finally, although liver involvement in brucellosis is usually manifested by a mild increase in transaminases, a frank cholestatic hepatitis may also occur and the patient may be subjected in unnecessary, time-consuming, costly and invasive interventions if Brucella is not included in the differential diagnosis.

\section{References}

1. Cuadrado A et al. Fever, pain in right hypochondrium and liver mass with central calcification. Revista española de enfermedades digestivas, 2004, 96(5):331-43.

2. Vallejo JG et al. Hepatosplenic abscesses due to Brucella melitensis: report of a case involving a child and review of the literature. Clinical infectious diseases, 1996, 22(3):485-9.

3. Kayacetin E, Turegen A, Saritas U. Hepatic abscess in brucellosis: a case report. Turkish journal of gastroenterology, 2003, 14(1):83-4.

4. Sadia Pérez D et al. [Brucella hepatic abscess. Report of a case and review of the literature] Absceso hepático por Brucella. Descripción de un caso y revisión de la literatura. Revista clínica española, 2001, 201:322-6.
5. Sisteron O et al. Hepatic abscess caused by Brucella US, CT and MRI findings: case report and review of the literature. Clinical imaging, 2002, 26:414-7.

6. Colmenero JD et al. Complications associated with Brucella melitensis infection: a study of 530 cases. Medicine (Baltimore), 1996, 75: 195-211.

7. Reihsaus E, Waldbaur H, Seeling W. Spinal epidural abscess: a meta-analysis of 915 patients. Neurosurgical review, 2000, 23(4):175-204.

8. Dagirmanjian A et al. MR imaging of vertebral osteomyelitis revisited. American journal of roentgenology, 1996, 167:1539.

9. Hendrick JW. Diagnosis and treatment of thyroiditis. Annals of surgery, 1956, 144:176-87. 
10. The endocrine system. In: Cotran RS, Kumar V, Robbins SL, eds. Robbins pathologic basis of disease, 5th ed. Philadelphia, WB Saunders, 1994:1125-8.

11. Park BW, Park CS. Pyriform sinus fistula. Yonsei medical journal, 1993, 34:386-90.

12. Barton GM et al. Combined Escherichia coli and Staphylococcus aureus thyroid abscess in an asymptomatic man. American journal of the medical sciences, 1988, 295:133-6.

13. Akdeniz $\mathrm{H}$ et al. Hematological manifestations in brucellosis cases in Turkey. Acta medica Okayama, 1998, 52:63-5.

14. Lynch EC, McKechnie JC, Alfrey Jr. CP. Brucellosis with pancytopenia. Annals of internal medicine, 1968, 69:319-22.

15. García P et al. Evaluación de la médula ósea en pacientes con brucelosis. Correlación clínicopathologica [Evaluation of the bone marrow in patients with brucellosis. Clinicopathologic correlation]. Enfermedades infecciosas y microbiología clínica, 1990, 8:19-24.
16. Al-Eissa Y et al. Pancytopenia in children with brucellosis: clinical manifestations and bone marrow findings. Acta haematological, 1993, 89:132-6.

17. Martin-Moreno SM et al. Pancytopenia due to haemophagocytosis in patients with brucellosis. A report of 4 cases. Journal of infectious diseases, 1983, 147:445-9.

18. Al-Eissa $\mathrm{Y}, \mathrm{Al}-\mathrm{Nasser} \mathrm{MN}$. Haematological manifestations of childhood brucellosis. Infection, 1993, 21:23-6.

19. Ariza J et al. Current understanding and management of chronic hepatosplenic suppurative brucellosis. Clinical infectious diseases, 2001, 32:1024-33.

20. Akritidis N, Pappas G. Ascites caused by brucellosis: a report of two cases. Scandinavian journal of gastroenterology, 2001, 36:110-2.

21. Kofteridis D et al. Human brucellosis in Crete, Greece. Poster 580. Clinical microbiology and infection, 2005, 11(Suppl. 2):159.

22. Akritidis $\mathrm{N}$ et al. The liver in brucellosis. Clinical gastroenterology and hepatology, 2007, 5(9):1109-12.

\section{Mediterranean Zoonoses Control Programme (MZCP) of the World Health Organization}

An inter-regional (Mediterranean and Middle East) Programme on zoonoses and foodborne disease prevention, surveillance and control through intersectoral collaboration and coordination.

The main objectives of the Programme: are

- $\quad$ promoting programmes for the prevention; surveillance \& control of zoonoses \& related foodborne diseases;

- $\quad$ strengthening collaboration between animals and public health services;

- implementing training activities;

- $\quad$ promoting veterinary public health activities and public health education;

- $\quad$ fostering collaboration among Member Countries.

Further information is available at: http://www.who.int/zoonoses/institutions/mzcp/en/ 